\title{
Charge-radius change and nuclear moments in the heavy tin isotopes from laser spectroscopy: Charge radius of ${ }^{132} \mathrm{Sn}$
}

\author{
F. Le Blanc, ${ }^{1}$ L. Cabaret, ${ }^{2}$ E. Cottereau, ${ }^{1}$ J. E. Crawford,${ }^{4}$ S. Essabaa, ${ }^{1}$ J. Genevey, ${ }^{5}$ R. Horn, ${ }^{3}$ \\ G. Huber, ${ }^{3}$ J. Lassen, ${ }^{3}$ J. K. P. Lee ${ }^{4}$ G. Le Scornet, ${ }^{6}$ J. Lettry, ${ }^{6}$ J. Obert, ${ }^{1}$ J. Oms, ${ }^{1}$ A. Ouchrif, ${ }^{1}$ \\ J. Pinard, ${ }^{2}$ H. Ravn, ${ }^{6}$ B. Roussière, ${ }^{1}$ J. Sauvage, ${ }^{1}$ and D. Verney ${ }^{1}$ \\ ${ }^{1}$ Institut de Physique Nucléaire, IN2P3-CNRS, F-91406 Orsay Cedex, France \\ ${ }^{2}$ Laboratoire Aimé Cotton, F-91405 Orsay Cedex, France \\ ${ }^{3}$ Institut für Physik der Universität Mainz, D-55099 Mainz, Germany \\ ${ }^{4}$ Physics Department, McGill University, Montréal, Canada H3A $2 T 8$ \\ ${ }^{5}$ Laboratoire de Physique Subatomique et Cosmologie, IN2P3-CNRS/Université Joseph Fourier, F-38026 Grenoble Cedex, France \\ ${ }^{6}$ CERN, 1211 Genève 23, Switzerland
}

(Received 25 March 2005; published 22 September 2005)

\begin{abstract}
Laser spectroscopy measurements have been carried out on the neutron-rich tin isotopes with the COMPLIS experimental setup. Using the $5 s^{2} 5 p^{2}{ }^{3} P_{0} \rightarrow 5 s^{2} 5 p 6 s{ }^{3} P_{1}$ optical transition, hyperfine spectra of ${ }^{126-132} \mathrm{Sn}$ and ${ }^{125,127,129-131} \mathrm{Sn}^{m}$ were recorded for the first time. The nuclear moments and the mean square charge radius variation $\left(\delta\left\langle r_{c}^{2}\right\rangle\right)$ were extracted. From the quadrupole moment values, these nuclei appear to be spherical. The magnetic moments measured are thus compared with those predicted by spherical basis approaches. From the measured $\delta\left\langle r_{c}^{2}\right\rangle$, the absolute charge radii of these isotopes were deduced in particular that of the doubly magic ${ }^{132} \mathrm{Sn}$ nucleus. The comparison of the results with several mean-field-type calculations have shown that dynamical effects play an important role in the tin isotopes.
\end{abstract}

DOI: 10.1103/PhysRevC.72.034305

PACS number(s): 21.10.Ft, 21.10.Ky, 27.60.+J, 31.30.Gs

\section{INTRODUCTION}

The doubly-magic nuclei are of great interest in nuclear physics because their properties (binding energy, radius ...) are the basis of the parametrization of the effective interactions used for mean-field calculations [1,2]. For the last two decades, these calculations [3-6] as well as the relativistic mean-field theory [7] successfully described the global properties of the nuclear ground states $[6,8,9]$. In parallel, a lot of new and accurate results were obtained providing systematic data available along isotopic series from light to heavy nuclei. This motivated many theoretical works in particular to improve the parameters of the effective interactions currently used. The goal of these theoretical studies is to define an effective interaction valid not only along the stability line but also for exotic nuclei. In the present work, we have studied the effect of the shell closure at $N=82$ on the mean square charge radius variation $\left(\delta\left\langle r_{c}^{2}\right\rangle\right)$ far from stability.

From the measurement of the isotope shift we have a direct access to the $\delta\left\langle r_{c}^{2}\right\rangle$ along isotope series. To perform such measurements on tin isotopes, we have used a technique consisting in ion-beam implantation followed by resonant ionization spectroscopy (RIS) studies of the laser desorbed radioactive atoms. Such a technique is applied to the COMPLIS system installed at the ISOLDE-Booster facility.

In this paper, we report on laser spectroscopy measurements performed on the heavy tin isotopes up to $A=132$. From the hyperfine spectra of ${ }^{126-132} \mathrm{Sn}$ as well of those of ${ }^{125,127,129-131} \mathrm{Sn}^{m}$, the variation of the mean square charge radius $\delta\left\langle r_{c}^{2}\right\rangle$ between these nuclei and the nuclear moments of the odd isotopes and their isomers were measured. These results have allowed us to determine the charge radius of the doubly-magic ${ }^{132} \mathrm{Sn}$ for the first time. They have shown that dynamical effects in the nucleus become important far from the neutron magic number.

\section{EXPERIMENTAL PROCEDURE}

\section{A. Isotope production}

The radioactive tin isotopes are produced via fission reactions in the ISOLDE $\mathrm{UC}_{2}$ target with the $1 \mathrm{GeV}$ CERN PS-Booster proton beam. This target can be associated either with the MK5 hot plasma ion source designed for the elements of low volatility or with a resonance ionization laser ion source (RILIS) tuned for tin. To determine the most suitable target-ion source system for laser spectroscopy measurements with COMPLIS, we have measured the isotope production of the two ion sources. In both cases, the ions extracted from the source and mass separated by the ISOLDE magnet are collected on a mylar/aluminum tape in front of a $\mathrm{Ge}(\mathrm{HP})$ detector in order to determine the number $N_{0}$ of the collected ions per second for each radioactive species in its ground and long-lived isomeric state. Figure 1 shows the $N_{0}$ values obtained with a $1 \mu \mathrm{C}$ proton pulse for the ground states of $\mathrm{Cd}, \mathrm{In}, \mathrm{Sb}, \mathrm{Te}$, and I with the MK5 source. In the case of tin, $N_{0}$ is shown for the ground and isomeric states using both ion sources. It is worth noting that with the MK5 ion source, many elements are ionized and for instance at $A=132$, tin represents only $0.24 \%$ of the observed nuclei. The $N_{0}$ values for $\mathrm{Cs}$ are difficult to obtain for $A \geqslant 132$ because of their long half-lives. However, this element is highly produced even with RILIS since it is easily ionized by the surface ionization mechanism. For instance at mass unit 130, Cs is produced 50 times more than tin with the hot plasma ion source and even 


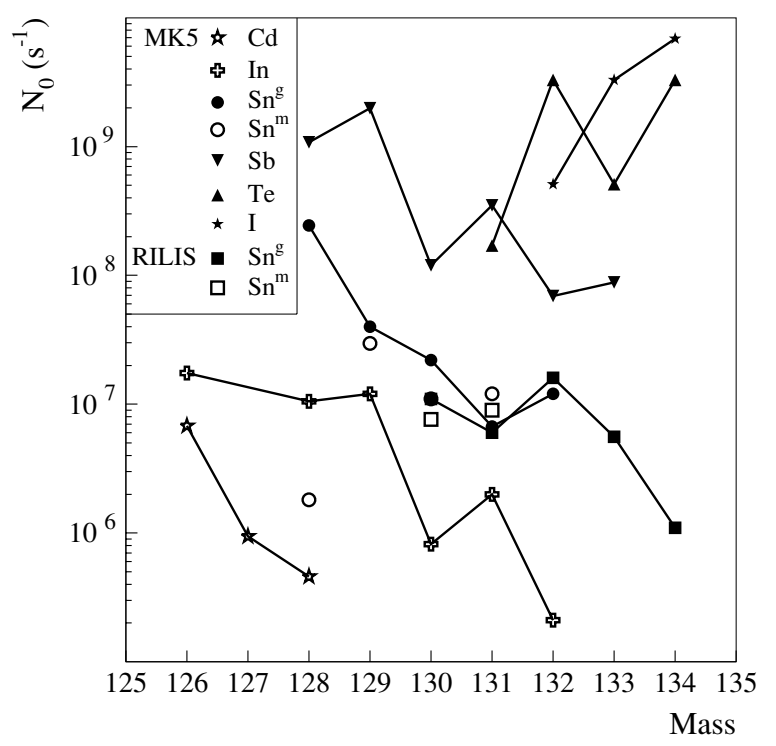

FIG. 1. Number of ions collected on the tape per second using the MK5 source for the ground states of $\mathrm{Cd}, \mathrm{In}, \mathrm{Sb}, \mathrm{Te}$, and I. For the tin isotopes, the $N_{0}$ values obtained using the MK5 and RILIS sources are indicated for the ground and isomeric states. The collection times ranged from 10 to $50 \mathrm{~ms}$ and there was no delay between the $1 \mu \mathrm{C}$ proton pulse and the collection.

more with RILIS. These various isobaric contaminations have to be taken into account to determine which ion source will be used to perform the laser spectroscopy measurements. Let us first recall the relationship between the $N_{0}$ values we have determined and the yield $Y$ commonly used at ISOLDE.

When the proton beam is continuously sent on the target, an equilibrium is obtained inside the target and $N_{0}$ represents the number of the studied atoms collected on the tape per time unit. $N_{0}$ is thus equal to $Y$ using a $1 \mu \mathrm{A}$ continuous proton beam. Since the $1 \mathrm{GeV}$ proton beam delivered by the CERN-PS-Booster is pulsed, $Y$ is now defined as the number of ions collected after one $1 \mu \mathrm{C}$ proton pulse [10]. It means that

$$
Y=\frac{N_{i}}{N_{p}} 6.2 \times 10^{12} \int_{0}^{+\infty} P(t) e^{-\lambda_{i} t} d t
$$

where $N_{i}$ is the number of atoms, corrected by the transport and ionization efficiency, that are generated per one proton pulse of $N_{p}$ protons and $P(t)$ the release function of the studied element. This quantity is the probability for a stable isotope created at $t=0$ to be released by the target at a time $t$. For a radioactive one, it becomes $P(t) e^{-\lambda_{i} t}, \lambda_{i}$ being its decay constant.

If $P(t)$ is known for the studied element, $Y$ can be extracted from $N_{0}$ knowing (i) the structure of the proton macrocycle (number of pulses and time between the pulses), (ii) the delay $\left(t_{1}\right)$ between the proton-beam impact and the beginning of the collection and (iii) the collection time $\left(t_{2}-t_{1}\right)$. When the half-life of the studied isotope is short $\left(T_{1 / 2} \leqslant 1 s\right)$, the relation
TABLE I. Calculated yields of some tin isotopes compared with the ISOLDE measured one.

\begin{tabular}{lcc}
\hline \hline$A$ & $\mathrm{Y}_{\text {from } N_{0}[1 / \mu \mathrm{C}]}$ & $\mathrm{Y}_{\text {ISOLDE }}[1 / \mu \mathrm{C}]$ \\
\hline 130 & $8.10^{8 \mathrm{a}}$ & $3.10^{8}$ \\
131 & $2.10^{8 \mathrm{a}}$ & $3.510^{8}$ \\
132 & $4.10^{8}$ & $3.10^{8}$ \\
133 & $2.10^{7}$ & $3.10^{7}$ \\
134 & $2.10^{6}$ & $2.10^{6}$ \\
\hline \hline
\end{tabular}

ancluding isomer production.

between $N_{0}$ and $Y$ becomes

$$
Y=\frac{N_{0}}{\lambda_{i}}\left(1-e^{-\lambda_{i}\left(t_{2}-t_{1}\right)}\right) \frac{\int_{0}^{+\infty} P(t) e^{-\lambda_{i} t} d t}{\int_{t_{1}}^{t_{2}} P(t) e^{-\lambda_{i} t} d t} .
$$

For the elements identified in the present work, only the $\mathrm{Sn}$ release function is known. The $Y$ values obtained for tin from our $N_{0}$ values are in quite good agreement with the yields measured by the ISOLDE group (see Table I) since, except for $A=130$, both determinations are equal within a factor of two. For the other elements observed, the release functions are not known so we cannot determine the corresponding ISOLDE yield. This is the reason we present our results in terms of $N_{0}$. These $N_{0}$ values allow us to draw a comparison between the various elements observed. They clearly indicate that the most perturbing elements for the laser spectroscopy measurement on tin should be the most produced ones, i.e., Te, I, and Cs (the latter being ionized with both ion sources). Cs is the element that brings the highest background and to minimize it, we chose the hot plasma source since its Cs yield is lower than that of the laser ion source while it is similar for tin.

\section{B. Experimental setup}

The ions are extracted at $60 \mathrm{kV}$ and mass separated by the High Resolution Separator (HRS) of ISOLDE. They enter the COMPLIS beam line and are slowed to $1 \mathrm{kV}$ to be deposited on the first atomic layers of a rotating graphite substrate. Once the amount of the collected atoms is optimum (following the half-life of the isotope to be studied) they are desorbed by a Nd:YAG laser beam and selectively ionized by a set of two pulsed, tunable dye lasers where the first excitation step at $286.3 \mathrm{~nm}\left(5 s^{2} 5 p^{2}{ }^{3} P_{0} \rightarrow 5 s^{2} 5 p 6 s^{3} P_{1}\right)$ is obtained from frequency doubling. The efficiency of the ionization step has been boosted using an auto-ionizing state at $413.627 \mathrm{~nm}$. The ions are finally detected with time-of-flight identification using a microchannel plate detector. The COMPLIS experimental setup is detailed in Ref. [11] and is shown in Fig. 2. The frequency scan is performed at the first excitation step and whenever the laser frequency corresponds to a hyperfine transition, the desorbed atoms are excited and ionized by the other fixed laser wavelengths.

For ${ }^{125-127} \mathrm{Sn}$ (longer lifetimes), the frequency scan over the hyperfine structure of a given isotope is made as follows: the implantation of the atoms is made over a wide band (the 


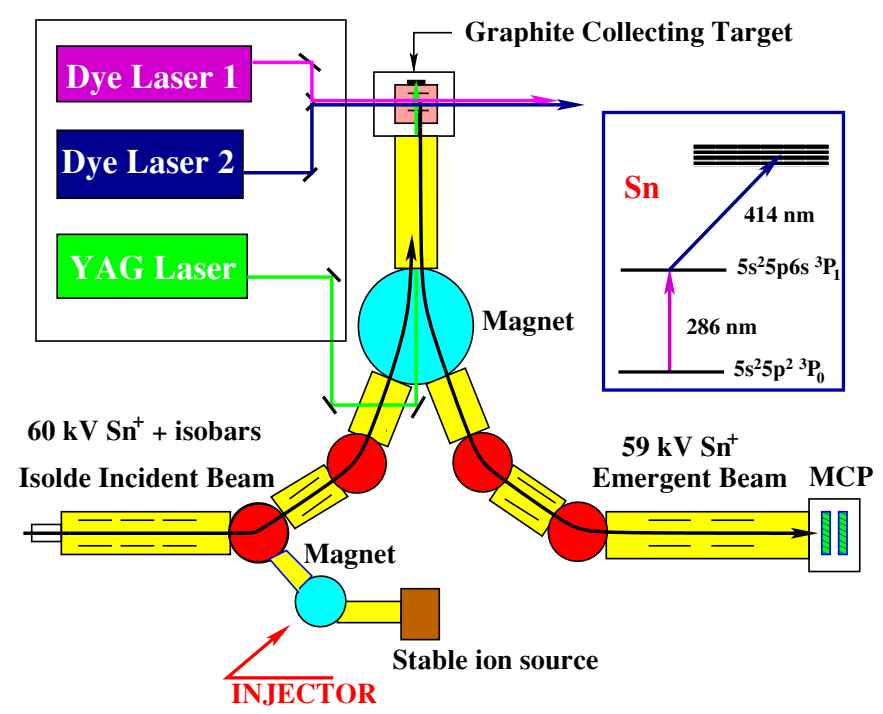

FIG. 2. (Color online) The COMPLIS experimental scheme used for tin.

target is slowly rotating). The target then goes back quickly to its origin and the desorption/ionization is performed at the same slow velocity all over this band and all over the hyperfine spectrum. The width of the band and the velocity are optimized as a function of the lifetime and of the production of the studied isotope. For the heavier nuclei (shorter lifetimes) the implantation is made as a single spot. After a sufficient collection time, the desorption of the tin atoms is made over the entire collection spot on the rotating target at a given excitation frequency. After the desorption is complete, a new cycle of implantation desorption is run at an advanced frequency step and all this is repeated over the whole hyperfine spectrum. The number of detected ions is directly proportional to the intensity of the hyperfine transition. With this apparatus, the efficiency we obtained is about $10^{-6}$. It was optimized using a stable beam ion injector as described in Ref. [12]. The best resolution obtained was about $170 \mathrm{MHz}$. We thus measured all the isotopes and isomers from $A=125$ to $A=132$. The hyperfine spectra of ${ }^{132} \mathrm{Sn},{ }^{131} \mathrm{Sn}^{g+m}$, and ${ }^{130} \mathrm{Sn}^{g+m}$ are shown as examples in Fig. 3. From the displacement of the centers of gravity of the hyperfine spectra, we were able to extract the isotope shift. The reference isotope used and also implanted with each isotope was ${ }^{126} \mathrm{Sn}$. From the relative position of the three lines of each isomer and odd isotope we have extracted the magnetic and quadrupole moments via the magnetic $A_{i}$ and electrostatic $B_{i}$ hyperfine constants. The frequency of each hyperfine line has been precisely measured using a $0.002 \mathrm{~cm}^{-1}$ band width lambda meter. To check the reliability of this apparatus, we have used, for each mass, the simultaneously recorded frequency of ${ }^{126} \mathrm{Sn}$.

\section{EXPERIMENTAL RESULTS}

The magnetic moments of the isomers and odd isotopes are obtained from $A_{i}$ of the atomic excited state and the precisely

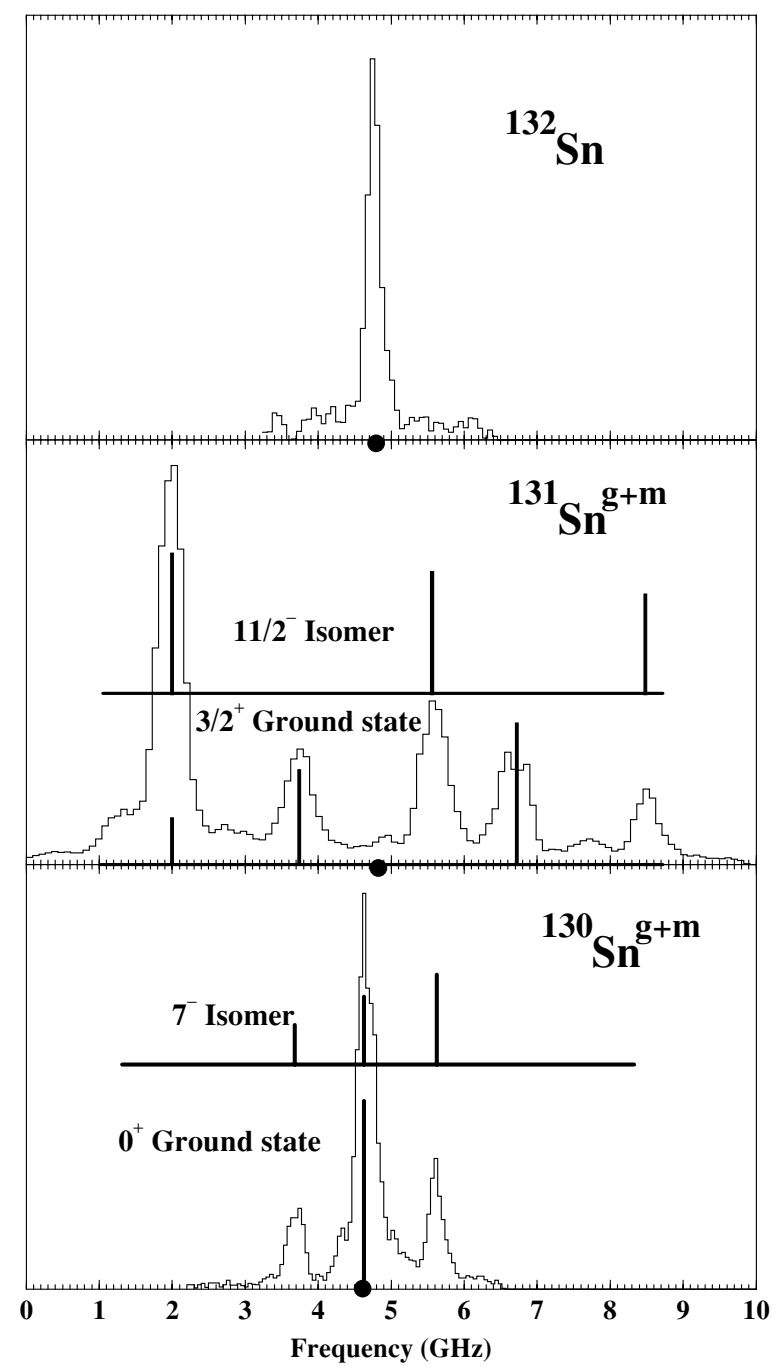

FIG. 3. Hyperfine spectra of ${ }^{130} \mathrm{Sn}^{g+m},{ }^{131} \mathrm{Sn}^{g+m}$, and ${ }^{132} \mathrm{Sn}$. For ${ }^{130} \mathrm{Sn}$ and ${ }^{131} \mathrm{Sn}$ one line of the isomer is mixed with one of the ground state.

known value of $\mu_{I}$ of ${ }^{119} \mathrm{Sn}$ corrected from diamagnetism [13]:

$$
\mu_{I}\left({ }^{x} \mathrm{Sn}\right)=0.4191(5) A_{i}\left({ }^{x} \mathrm{Sn}\right) I\left({ }^{x} \mathrm{Sn}\right)\left[\mu_{N}\right],
$$

with $A_{i}$ in $\mathrm{GHz}$. The hyperfine anomaly can be neglected with respect to experimental uncertainties (see [13]). The $\mu_{I}$ values are presented in Table II. The value we measured for

TABLE II. Nuclear moments of the isomers and odd $\mathrm{Sn}$ isotopes.

\begin{tabular}{lcrrcc}
\hline \hline$A$ & $I^{\pi}$ & $A_{i}[\mathrm{GHz}]$ & $\mu_{I}\left[\mu_{N}\right]$ & $B_{i}[\mathrm{GHz}]$ & $Q_{s}[\mathrm{~b}]$ \\
\hline $125^{g}$ & $11 / 2^{-}$ & $-0.585(2)$ & $-1.348(6)$ & $-0.02(3)$ & $+0.14(21)$ \\
$125^{m}$ & $3 / 2^{+}$ & $1.216(5)$ & $+0.764(3)$ & & \\
$127^{g}$ & $11 / 2^{-}$ & $-0.577(3)$ & $-1.329(7)$ & $-0.041(18)$ & $+0.30(13)$ \\
$127^{m}$ & $3 / 2^{+}$ & $1.205(6)$ & $+0.757(4)$ & & \\
$129^{g}$ & $3 / 2^{+}$ & $1.200(5)$ & $+0.754(3)$ & & \\
$129^{m}$ & $11 / 2^{-}$ & $-0.563(2)$ & $-1.297(5)$ & $0.025(24)$ & $-0.18(17)$ \\
$130^{m}$ & $7^{-}$ & $-0.130(1)$ & $-0.381(3)$ & $0.050(15)$ & $-0.36(11)$ \\
$131^{g}$ & $3 / 2^{+}$ & $1.188(7)$ & $+0.747(4)$ & & \\
$131^{m}$ & $11 / 2^{-}$ & $-0.554(2)$ & $-1.276(5)$ & $-0.003(28)$ & $+0.02(20)$ \\
\hline \hline
\end{tabular}


TABLE III. Isotope shift, $\delta\left\langle r_{c}^{2}\right\rangle$ relative to ${ }^{120} \mathrm{Sn}$ and absolute charge radii.

\begin{tabular}{llcc}
\hline \hline$A$ & $I S[\mathrm{GHz}]$ & $\delta\left\langle r_{c}^{2}\right\rangle^{120, A}\left[\mathrm{fm}^{2}\right]$ & $r_{c}[\mathrm{fm}]$ \\
\hline $125^{g}$ & $0.503(11)$ & $0.236(31)$ & $4.677(3)$ \\
$125^{m}$ & $0.455(8)$ & $0.221(30)$ & $4.676(3)$ \\
126 & $0.63(1)$ & $0.290(37)$ & $4.683(4)$ \\
$127^{g}$ & $0.685(10)$ & $0.322(42)$ & $4.687(4)$ \\
$127^{m}$ & $0.622(10)$ & $0.302(41)$ & $4.685(4)$ \\
128 & $0.801(12)$ & $0.373(48)$ & $4.692(4)$ \\
$129^{g}$ & $0.794(11)$ & $0.384(52)$ & $4.693(5)$ \\
$129^{m}$ & $0.881(9)$ & $0.411(53)$ & $4.696(6)$ \\
$130^{g}$ & $1.006(10)$ & $0.465(59)$ & $4.702(6)$ \\
$130^{m}$ & $0.906(8)$ & $0.434(63)$ & $4.699(6)$ \\
$131^{g}$ & $1.141(12)$ & $0.521(66)$ & $4.708(7)$ \\
$131^{m}$ & $1.226(10)$ & $0.548(67)$ & $4.711(7)$ \\
132 & $1.140(6)$ & $0.534(69)$ & $4.709(7)$ \\
\hline \hline
\end{tabular}

${ }^{125} \mathrm{Sn}^{g}$ is in perfect agreement with that obtained by Anselment et al. [14] $\left(\mu_{I}=-1.348(2) \mu_{N}\right)$.

Also, for the isomers and the odd isotopes, the $B_{i}$ factors of the ${ }^{3} P_{1}$ atomic excited state have been extracted from the hyperfine spectra. $Q_{s}$ is related to $B_{i}$ via

$$
Q_{s}\left({ }^{x} \mathrm{Sn}\right)=-7.25 B_{i}\left({ }^{x} \mathrm{Sn}\right)[b]
$$

which is obtained from the calculated electric field gradient as described in Ref. [13]. The measured $Q_{s}$ values are presented in Table II except for the $3 / 2^{+}$states for which the weakest line was buried in the strongest component of the $11 / 2^{-}$state and could thus not be isolated.

The experimental isotope shift consists of a mass shift $\delta v_{\mathrm{MS}}$ and a field shift $\delta v_{\mathrm{FS}}$; it is from this latter contribution that $\delta\left\langle r_{c}^{2}\right\rangle$ beween two nuclei $A$ and $A^{\prime}$ can be extracted. The isotope shift can thus be expressed as

$$
\delta v^{A, A^{\prime}}\left(\frac{A A^{\prime}}{A^{\prime}-A}\right)=K F_{\lambda} \delta\left\langle r_{c}^{2}\right\rangle^{A, A^{\prime}}\left(\frac{A A^{\prime}}{A^{\prime}-A}\right)+M,
$$

where $K=0.975$ (deduced from Ref. [15]).

To evaluate $F_{\lambda}$ and $M$ we have performed a King Plot taking into account the error bars on both axes using a linear fit

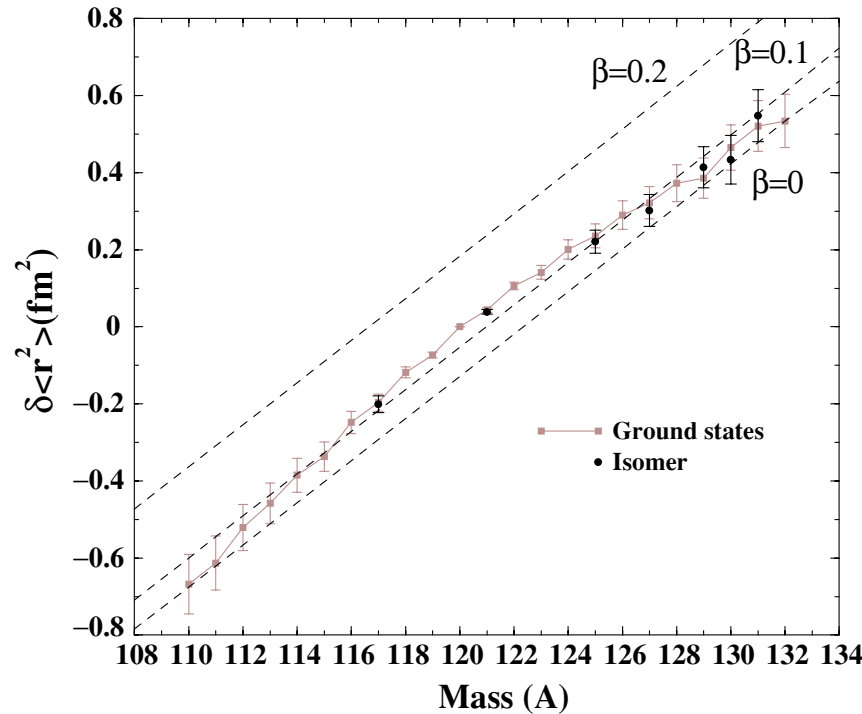

FIG. 4. (Color online) Charge radius change in the tin isotopes $([14,17]$ and this measurement). The line joins all the ground state nuclei. Also are shown the isodeformation lines calculated from the droplet model [19].

program as described in Ref. [16]. This King Plot is made with the isotope shift measured in Ref. [14] versus the muonic $\delta\left\langle r_{c}^{2}\right\rangle$ measured in Ref. [17] including all isotope pair combinations as described in Ref. [18]. The result of the fit gives $F_{\lambda}=$ $3.30(27) \mathrm{GHz} / \mathrm{fm}^{2}$ and $M=-761(200) \mathrm{GHz}$. The $\delta\left\langle r_{c}^{2}\right\rangle$ values are presented in Table III. Knowing the value of $r_{c}\left({ }^{120} \mathrm{Sn}\right)$ from Ref. [17] we have deduced the values of the absolute radii that are also shown in Table III. The $\delta\left\langle r_{c}^{2}\right\rangle$ curve obtained with these new $F$ and $M$ values is presented Fig. 4.

\section{DISCUSSION}

\section{A. Nuclear Moments}

All the quadrupole moments we measured are quite small. If we assume axial symmetry, one can extract the $\beta$ deformation. The results are presented in Table IV. The $\beta$ values are very

TABLE IV. Comparison between experimental and calculated magnetic moments of the isomers and odd $\mathrm{Sn}$ isotopes. The spectroscopic quadrupole moments are also shown as well as the $\beta$ deformation assuming axial

\begin{tabular}{|c|c|c|c|c|c|c|c|}
\hline$A$ & $I^{\pi}$ & State & $\mu_{\exp }\left[\mu_{N}\right]$ & $\mu_{\text {cal }}{ }^{\mathrm{a}}\left[\mu_{N}\right]$ & $\mu_{\text {cal }}{ }^{\mathrm{b}}\left[\mu_{N}\right]$ & $Q_{s}[\mathrm{~b}]$ & $\beta$ \\
\hline $125^{g}$ & $11 / 2^{-}$ & $1 h_{11 / 2}$ & $-1.348(6)$ & -1.913 & -1.225 & $+0.14(21)$ & $+0.02(3)$ \\
\hline $125^{m}$ & $3 / 2^{+}$ & $2 d_{3 / 2}$ & $+0.764(3)$ & 1.148 & 0.831 & & \\
\hline $127^{g}$ & $11 / 2^{-}$ & $1 h_{11 / 2}$ & $-1.329(7)$ & -1.913 & -1.225 & $+0.30(13)$ & $+0.04(2)$ \\
\hline $127^{m}$ & $3 / 2^{+}$ & $2 d_{3 / 2}$ & $+0.757(4)$ & 1.148 & 0.831 & & \\
\hline $129^{g}$ & $3 / 2^{+}$ & $2 d_{3 / 2}$ & $+0.754(3)$ & 1.148 & 0.831 & & \\
\hline $129^{m}$ & $11 / 2^{-}$ & $1 h_{11 / 2}$ & $-1.297(5)$ & -1.913 & -1.225 & $-0.18(17)$ & $-0.02(2)$ \\
\hline $130^{m}$ & $7^{-}$ & $2 d_{3 / 2} \otimes 1 h_{11 / 2}$ & $-0.381(3)$ & -0.770 & -0.394 & $-0.36(11)$ & $-0.04(2)$ \\
\hline $131^{g}$ & $3 / 2^{+}$ & $2 d_{3 / 2}$ & $+0.747(4)$ & 1.148 & 0.831 & & \\
\hline $131^{m}$ & $11 / 2^{-}$ & $1 h_{11 / 2}$ & $-1.276(5)$ & -1.913 & -1.225 & $+0.02(20)$ & $+0.002(20)$ \\
\hline
\end{tabular}
symmetry.

${ }^{\mathrm{a}} g_{s}=g_{s, \text { free }}$ and $g_{l}=0$

${ }^{\mathrm{b}} g_{s}=-2.65$ and $g_{l}=0.02$ 


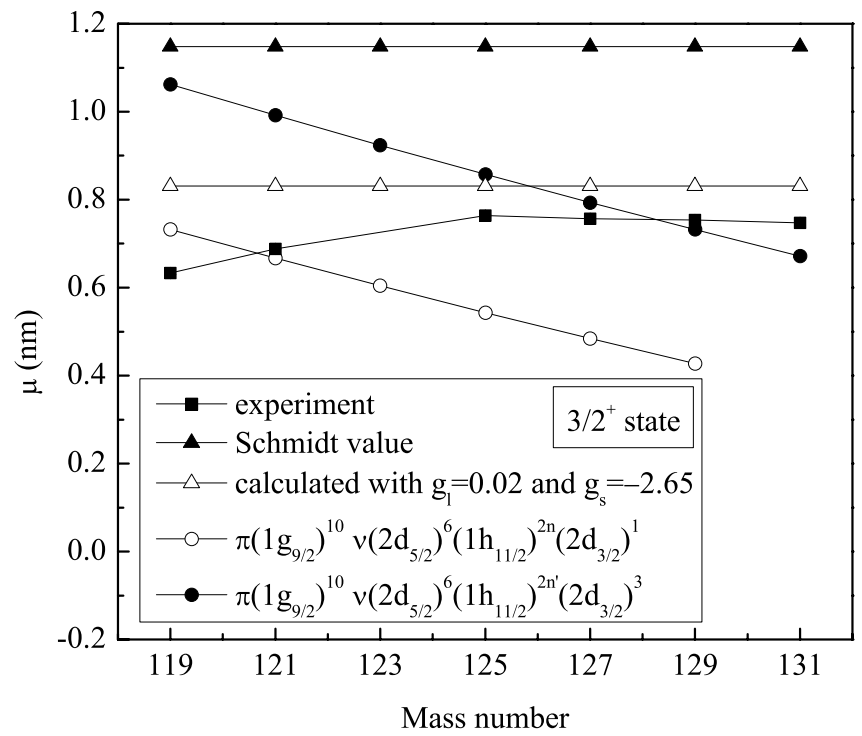

FIG. 5. Evolution of the magnetic moment as a function of the mass for the $3 / 2^{+}$state and comparison with several calculations. The value of the $V_{s} I$ quantity defined in Refs. [21,22] and used for the calculation is $-25 / A \mathrm{MeV}$. $\mu_{\exp }(119)$ is taken from [23] and $\mu_{\exp }(121)$ from [14].

weak which confirms a spherical shape for all these magic nuclei.

One can compare the experimental values of the corresponding $\mu_{I}$ with the calculated ones using the Schmidt model with $g_{l}=0$ and $g_{s}=g_{s, \text { free }}=-3.826$. This is reported in column 5 of Table IV. If we just consider this simple calculation one can now settle the spin values between $3 / 2$ and $11 / 2$ for each state, values uncertain up to now. However, these calculated $\mu_{I}$ values differ from the measured ones by 40 to $50 \%$. In this mass region, $g_{l}=0.02$ and $g_{s}=-2.65$ can be chosen to give a good estimate of the $g$ factors measured for the $2 d_{3 / 2}$ and the $1 h_{11 / 2}$ neutron states of ${ }^{135} \mathrm{Xe}$. This globally takes into account the core polarization and meson-exchange current effects on the single-particle $g$ factors [20]. The calculated $\mu_{I}$ using these $g$ values are reported in column 6 of Table IV. They differ from the measured ones only by about $10 \%$. We also represent in Figs. 5 and 6 the evolution of the magnetic moments as the function of the mass for $1 h_{11 / 2}$ and $2 d_{3 / 2}$. It clearly shows the increase of $\mu_{I}$ with $A$ under the line $g_{l}=0.02$ and $g_{s}=-2.65$ which could indicate a small collective contribution in the $g$ values related to configuration mixing.

In the frame of the theoretical approach developed by Arima, Horie and Noya [21,22], we have estimated the deviation of the magnetic moments from the Schmidt values taking into account configuration mixing. To contribute to the magnetic moment, the excited configuration that can mix with the ground state must have an intermediate angular momentum equal to 1 . In this way, nucleons in the $j_{1}=l_{1}+1 / 2$ orbit cannot play a role in the deviation of $\mu_{I}$ when the $j_{2}=$ $l_{1}-1 / 2$ orbit is fully occupied. Indeed, obviously in this case, the $j_{1}$ orbit is filled too. In the following, for sake of simplicity, we indicate only the components of the configurations that play

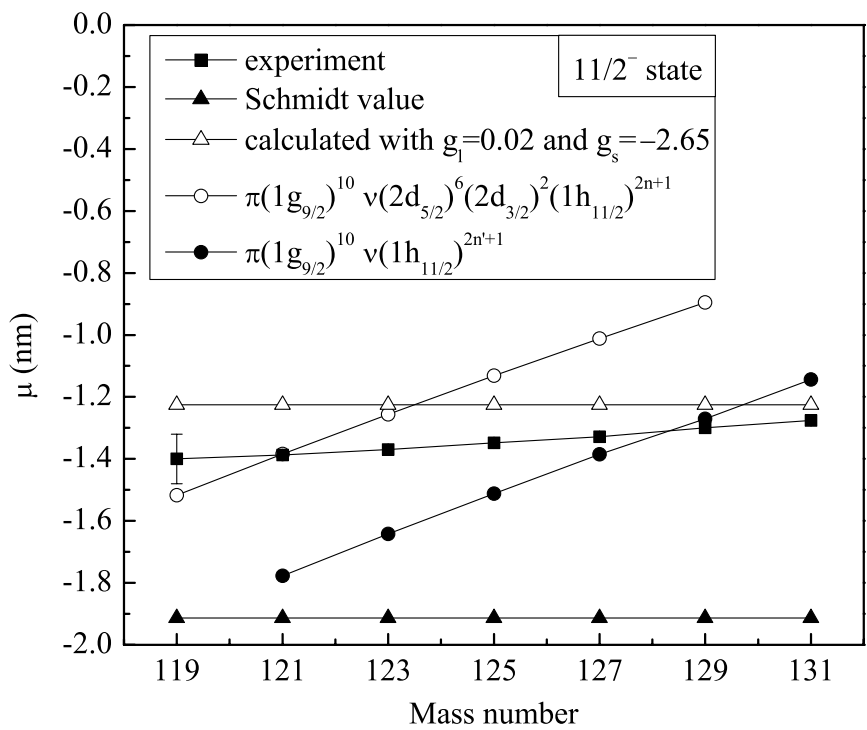

FIG. 6. Evolution of the magnetic moment as a function of the mass for the $11 / 2^{-}$state and comparison with several calculations. The value of $V_{s} I$ has been taken to be $-15 / A \mathrm{MeV}$. $\mu_{\exp }(119)$ is taken from Ref. [24] and $\mu_{\text {exp }}(121,123)$ from Ref. [14].

a role in the calculated $\mu_{I}$ values. For the protons, only the $\left(1 g_{9 / 2}\right)^{10}$ component appears because the other orbits of the 28 and 50 shells $\left(2 p_{3 / 2}, 1 f_{5 / 2}\right.$, and $\left.2 p_{1 / 2}\right)$ have a fully occupied $j$-conjugate orbital. Thus, the configurations used to describe the $3 / 2^{+}$and $11 / 2^{-}$states in the tin isotopes are

(i) $\left(1 g_{9 / 2}\right)^{10}$ for protons and $\left(2 d_{5 / 2}\right)^{6}\left(1 h_{11 / 2}\right)^{2 n}\left(2 d_{3 / 2}\right)^{1}$ or $\left(2 d_{5 / 2}\right)^{6}\left(1 h_{11 / 2}\right)^{2 n^{\prime}}\left(2 d_{3 / 2}\right)^{3}$ for neutrons

(ii) $\left(1 g_{9 / 2}\right)^{10}$ for protons and $\left(2 d_{5 / 2}\right)^{6}\left(2 d_{3 / 2}\right)^{2}\left(1 h_{11 / 2}\right)^{2 n+1}$ or $\left(1 h_{11 / 2}\right)^{2 n^{\prime}+1}$ for neutrons, respectively.

The results of the calculation are shown in Figs. 5 and 6. In addition to the configuration mixing described in Refs. [21,22], an admixture between the two proposed neutron configurations seems to be necessary to account for the experimental values from ${ }^{119} \mathrm{Sn}$ to ${ }^{131} \mathrm{Sn}$.

The $\mu_{I}$ values reported for the $7^{-}$state of ${ }^{130} \mathrm{Sn}$ have been calculated using the additivity relation [25]:

$$
\begin{array}{r}
\mu_{I}=\frac{I}{2}\left[\left(g_{1}+g_{2}\right)+\left(g_{1}-g_{2}\right)\left(\frac{j_{1}\left(j_{1}+1\right)-j_{2}\left(j_{2}+1\right)}{I(I+1)}\right)\right] \\
\text { with } \quad g_{i}=g_{l} \pm \frac{g_{s}^{\text {eff }}-g_{l}}{2 l_{i}+1} ; \quad j_{i}=l_{i} \pm 1 / 2 .
\end{array}
$$

Also for ${ }^{130} \mathrm{Sn}$, the agreement between the prediction and the experimental value is clearly improved when $g_{l}=0.02$ and $g_{s}=-2.65$ (see Table IV).

\section{B. Charge radii}

The mean square charge radius variation from $A=108$ to $A=132$ is shown in Fig. 4 together with the $\delta\left\langle r_{c}^{2}\right\rangle$ behavior expected from the droplet model [19] assuming different deformation parameters, namely $\beta=0,0.1$, and 0.2 . Our experimental $\delta\left\langle r_{c}^{2}\right\rangle$ between $A=125$ and $A=132$ confirm 


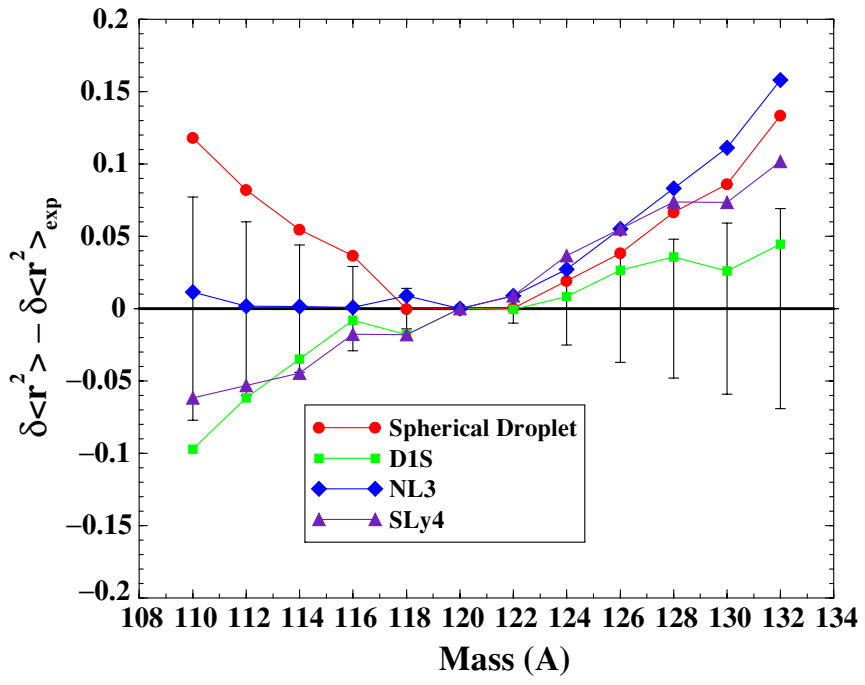

FIG. 7. (Color online) Difference between the calculated $\delta\left\langle r_{c}^{2}\right\rangle$ and the experimental ones for several mean field static models.

the parabolic trend observed below mass 125. This curve can be compared with that predicted in the framework of microscopic approaches as (i) the relativistic mean field theory (RMF+BCS) using the NL3 parameter set and constant pairing gaps [26], (ii) the Hartree-Fock-Bogolyubov mean-field (HFB) with the D1S Gogny force [2,27,28], and (iii) the HFB mean-field with the SLy4 effective interaction and delta type pairing force [29,30]. All the predicted $\delta\left\langle r_{c}^{2}\right\rangle$ values are almost similar to the experimental ones. To show up their different behaviors, we have reported in Fig. 7 the difference between the experimental and the calculated values for each model with $A=120$ as the reference mass. For the RMF-NL3 model, theoretical and experimental results agree for the light masses and disagree for $A>124$. For HFB-D1S calculations, the agreement is good for the heavy masses and rather bad for $A<114$. The HFB-SLy4 treatment yields intermediate results.

From our $\delta\left\langle r_{c}^{2}\right\rangle$ measurement and the ${ }^{120} \mathrm{Sn}\left\langle r_{c}\right\rangle$ value of Ref. [17], we obtain the charge radius of the doubly-magic ${ }^{132}$ Sn nucleus: $\left\langle r_{c}\right\rangle=4.709(7) \mathrm{fm}$. It is worth noting that this value is well reproduced by all of the theoretical approaches considered previously:

$\left\langle r_{c}\right\rangle=4.710 \mathrm{fm}$ with RMF-NL3,

$\left\langle r_{c}\right\rangle=4.693 \mathrm{fm}$ with HFB-D1S,

$\left\langle r_{c}\right\rangle=4.704 \mathrm{fm}$ with HFB-SLy4

We have also to note that a charge radius $\left\langle r_{c}\right\rangle=4.71 \mathrm{fm}$, in perfect agreement with the experimental value, was predicted as early as 1974 by Hartree-Fock+BCS calculations [31].

In Fig. 7, the disagreement observed for $A=132$ results of course of the choice of the reference mass $A=120$. That has been done because the measured value was precise and also because it is right in the middle of the measured ones. In Fig. 8 we compare the measured $\left\langle r_{c}\right\rangle\left({ }^{120} \mathrm{Sn}\right)(4.652 \mathrm{fm})$ with the ones predicted by the three static approaches considered above and also with those obtained when dynamical effects are taken into account: Microscopic Bohr Hamiltonian (HFB-D1S) [32,33]

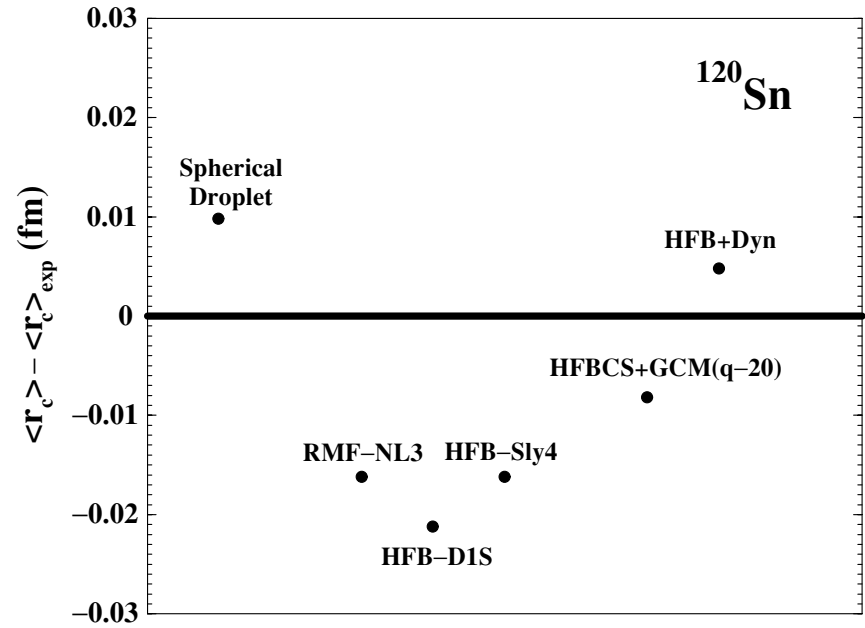

FIG. 8. Difference between the experimental $\left\langle r_{c}\right\rangle$ of ${ }^{120} \mathrm{Sn}$ and the calculated ones for several mean field static and dynamic models.

and generator coordinate method (HFB-Sly4) [30]. These two approaches are configuration mixing calculations in variational spaces built with constrained HFB (with D1S or SLy4) states covering quadrupole axial and triaxial deformations.

It is very clear that the ${ }^{120} \mathrm{Sn}\left\langle r_{c}\right\rangle$ value is much better reproduced when dynamical effects are taken into account. Thus, one can conclude that the parabolic shape observed in Fig. 4 is very likely due to important dynamical effects in the Sn magic isotope series as previously mentioned in Refs. [30] and [34].

\section{CONCLUSION}

Hyperfine structure splitting and isotope shift have been measured for the neutron rich tin isotopes from $A=125$ to $A=132$ by resonance ionization spectroscopy on an atomic beam produced by laser desorption. Such measurements have been successful in spite of the very low proportion of tin in the ISOLDE isobaric beam showing that COMPLIS is well adapted to perform such studies with a contaminated beam. The quadrupole moments of the $11 / 2^{-}$states as well as that of ${ }^{130} \mathrm{Sn}^{m}$ have been measured showing a spherical shape for all these nuclei. The magnetic moments of all the odd isotopes and of all the isomers were measured and compared with those predicted by spherical model and other approaches pointing out some configuration mixing in all the studied nuclei. Thanks to measurements made at high resolution, the radius of ${ }^{132} \mathrm{Sn}$ has been precisely measured. The parabolic shape of the charge radius variation previously observed has been extended up to mass 132. These results have been compared with several mean-field models and have shown that dynamical effects play an important role in tin and deforms the nucleus as one gets far from the magic neutron shell. However, the last question to address is about the variation of the mean square charge radius beyond $A=132$. Is there a kink at the crossing of the magic shell? The measurement of the $\delta\left\langle r_{c}^{2}\right\rangle$ at $A=134$ would answer this question. 
[1] D. Vautherin and D. M. Brink, Phys. Rev. C 5, 626 (1972).

[2] J. Dechargé and D. Gogny, Phys. Rev. C 21, 1568 (1980); J. F. Berger, M. Girod, and D. Gogny, Nucl. Phys. A428, 23c (1984) .

[3] P. Quentin and H. Flocard, Annu. Rev. Nucl. Sci. 28, 523 (1978).

[4] M. Girod and B. Grammaticos, Phys. Rev. C 27, 2317 (1983).

[5] M. Girod, J. P. Delaroche, D. Gogny, and J. F. Berger, Phys. Rev. Lett. 62, 2452 (1989).

[6] Z. Patyk, A. Baran, J. F. Berger, J. Dechargé, J. Dobaczewski, P. Ring, and A. Sobiczewski, Phys. Rev. C 59, 704 (1999).

[7] J. D. Walecka, Ann. Phys. (NY) 83, 491 (1974).

[8] P.-G. Reinhard, Rep. Prog. Phys. 52, 439 (1989).

[9] V. Blum, J. A. Maruhn, P.-G. Reinhard, and W. Greiner, Phys. Lett. B323, 262 (1994).

[10] J. Lettry, R. Catherall, P. Drumm, P. Van Duppen, A. H. M. Evensen, G. J. Focker, A. Jokinen, O. C. Jonsson, E. Kugler, H. Ravn, and ISOLDE Collaboration, Nucl. Instrum. Methods Phys. Res. B 126, 130 (1997).

[11] J. Sauvage et al., Hyp. Int. 129, 303 (2000).

[12] D. Verney et al., submitted to Eur. Phys. J. A (2004).

[13] J. Eberz, U. Dinger, G. Huber, H. Lochmann, R. Menges, G. Ulm, R. Kirchner, O. Klepper, T. U. Kühl, and D. Marx, Z. Phys. A 326, 121 (1987).

[14] M. Anselment, K. Bekk, A. Hanser, H. Hoeffgen, G. Meisel, S. Göring, H. Rebel, and G. Schatz, Phys. Rev. C 34, 1052 (1986).

[15] G. Torbohm, B. Fricke, and A. Rosén, Phys. Rev. A 312038 (1985).

[16] P. H. Borcherds and C. V. Smith, Eur. J. Phys. 16, 204 (1995).
[17] C. Piller, C. Gugler, R. Jacot-Guillarmod, L. A. Schaller, L. Schellenberg, H. Schneuwly, G. Fricke, T. Hennemann, and J. Herberz, Phys. Rev. C 42, 182 (1990).

[18] W. Neu, G. Passler, G. Sawatzky, R. Winkler, and H.-J. Kluge, Z. Phys. D 7, 193 (1987).

[19] W. Myers and W. Swiatecki, Nucl. Phys. A410, 61 (1983).

[20] G. Jakob et al., Phys. Rev. C 65, 024316 (2002).

[21] A. Arima and H. Horie, Prog. Theor. Phys. 12, 623 (1954).

[22] H. Noya, A. Arima, and H. Horie, Prog. Theor. Phys. suppl. 8, 33 (1958).

[23] G. Crecelius, Z. Phys. 258, 56 (1973).

[24] D. F. Gumprecht, T. E. Katila, L. C. Moberg, and P. O. Lipas, Phys. Lett. A40, 297 (1972).

[25] K. Krien, B. Klemme, R. Folle, and E. Bodenstedt, Nucl. Phys. A228, 15 (1974).

[26] G. A. Lalazissis, S. Raman, and P. Ring, At. Data Nucl. Data Tables 71, 1 (1999).

[27] J. Dechargé, M. Girod, and D. Gogny, Phys. Lett. B55, 361 (1975).

[28] S. Peru (private communication) (1998).

[29] E. Chabanat, P. Bonche, P. Haensel, J. Meyer, and R. Shaeffer, Nucl. Phys. A627, 710 (1997); A635, 231 (1998); A643, 441 (1998).

[30] K. Bennaceur, Paul Bonche, and J. Meyer, C. R. Phys. 4, 555 (2003).

[31] M. Beiner and R. Lombard, Annu. Phys. (NY) 86, 262 (1974).

[32] J. Libert, M. Girod, and J.-P. Delaroche., Phys. Rev. C 60, 054301 (1999).

[33] J. Libert and M. Girod (private communication) (2001).

[34] J. Sauvage et al., IPNO annual report No. 32 (2001). 